\title{
Coordinated Millimeter Wave Beam Selection Using Fingerprint for Cellular- Connected Unmanned Aerial Vehicle
}

\author{
Sangmi Moon ${ }^{1}$, Hyeonsung Kim², Young-Hwan You ${ }^{3}$, Cheol Hong Kim ${ }^{4}$ and Intae Hwang ${ }^{5 *}$ \\ ${ }^{1}$ Department of IT Artificial Intelligence, Korea Nazarene University, \\ Wolbong-ro 48, Cheonan-city, Choongcheongnam-do, 31172, Republic of Korea \\ moonsm@kornu.ac.kr \\ ${ }^{2}$ Department of Electronic Engineering, Chonnam National University, \\ 77, Yongbong-ro, Buk-gu, Gwangju, 61186, Republic of Korea \\ hsk940127@naver.com \\ ${ }^{3}$ Department of Computer Engineering, Sejong University \\ 209, Neungdong-ro, Gwangjin-gu, Seoul, 05006, Republic of Korea \\ [yhyou@sejong.ac.kr] \\ ${ }^{4}$ School of Computer Science and Engineering, Soongsil University, \\ 369 Sangdo-Ro, Dongjak-Gu, Seoul, 06978, Republic of Korea \\ [cheolhong@ssu.ac.kr] \\ ${ }^{5}$ Department of Electronic Engineering and Department of ICT Convergence System Engineering, \\ College of Engineering, Chonnam National University, \\ 77, Yongbong-ro, Buk-gu, Gwangju, 61186, Republic of Korea \\ [e-mail: hit@jnu.ac.kr] \\ ${ }^{*}$ Corresponding author: Intae Hwang
}

Received February 7, 2021; revised March 19, 2021; accepted March 30, 2021; published May 31, 2021

\begin{abstract}
Millimeter wave (mmWave) communication based on the wide bandwidth of $>28 \mathrm{GHz}$ is one of the key technologies for cellular-connected unmanned aerial vehicles (UAVs). The selection of mmWave beams in such cellular-connected UAVs is challenging and critical, especially when downlink transmissions toward aerial user equipment (UE) suffer from poor signal-to-interference-plus-noise ratio (SINR) more often than their terrestrial counterparts. This study proposed a coordinated mmWave beam selection scheme using fingerprint for cellular-connected UAV. The scheme comprises fingerprint database configuration and coordinated beam selection. In the fingerprint database configuration, the best beam index from the serving cell and interference beam indexes from neighboring cells are stored. In the coordinated beam selection, the best and interference beams are determined using the

This research was supported by the Ministry of Science and ICT (MSIT), Korea, under the Information Technology Research Center (ITRC) support program (IITP-2020-2016-0-00314) supervised by the Institute for Information \& communications Technology Planning \& Evaluation (IITP). This work was supported by the National Research Foundation of Korea (NRF) grant funded by the Korea government (MSIT: Ministry of Science and ICT) (2020R1I1A1A01073948 and 2021R1A2C1005058). This research was also supported by the BK21 FOUR Program(Fostering Outstanding Universities for Research, 5199991714138) funded by the Ministry of Education(MOE, Korea) and National Research Foundation of Korea(NRF).
\end{abstract}


fingerprint database information instead of performing an exhaustive search, and the coordinated beam transmission improves the SINR for aerial UEs. System-level simulations assess the UAV effect based on the third-generation partnership project-new radio mmWave and UAV channel models. Simulation results show that the proposed scheme can reduce the overhead of exhaustive search and improve the SINR and spectral efficiency.

Keywords: Coordinated beam selection, fingerprint, mmWave, UAV.

\section{Introduction}

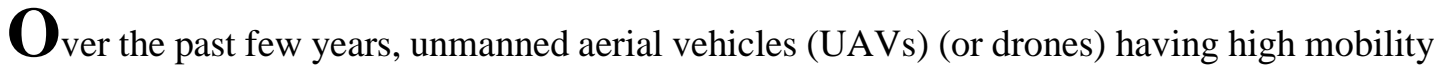
are used in various fields [1], [2]. Earlier, UAVs were mainly deployed in hostile areas by the military to reduce pilot loss; however, in recent years, UAVs have been used for weather observation, natural disaster detection, traffic control, and cargo transportation as they have been miniaturized and lightened and are inexpensive [3]. In addition, UAV is a representative convergence field in which next-generation technologies, e.g., aircraft technology, communication/network, sensors, and artificial intelligence, are combined. Therefore, it is possible to create various use cases, such as logistics and infrastructure management, based on the high-speed movement of UAVs and non-visible flights using cellular communications, which support broadband and mobility.

As 3GPP evolved to 3G WCDMA and 4G LTE, it has expanded the service range, which includes data traffic centricity, broadband, high-speed mobility, and QoS support. With 5G standardization, the concept of providing communication services to people and objects on the terrestrial was expanded, and a new study item for cellular communication standard technology research for UAVs as aerial user equipment (UE), known as cellular-connected UAVs, was concluded [4]. Cellular-connected communications, which can significantly improve UAV-terrestrial communication, use terrestrial base stations (BSs) of the cellular network [5]-[7]. In this network, aerial UEs receive line-of-sight (LoS) signals from numerous terrestrial BSs as their altitude increases [8]. Thus, aerial UEs can receive significant interference from numerous terrestrial BSs transmitting toward the aerial and terrestrial UEs. As a result, downlink transmissions toward aerial users have lower signal-to-interference-plusnoise ratios (SINRs) than terrestrial UEs.

Millimeter wave (mmWave) communication with a wide bandwidth of $>28 \mathrm{GHz}$ is one of the key technologies for high-speed UAV communications [9]. Although mmWave communication for 5G and cellular systems on the ground has been extensively studied, the application of mmWave communication to cellular-connected UAV systems in an aerial scenario has opportunities and challenges. Meanwhile, the mmWave signal is vulnerable to obstacles; therefore, the UAV-terrestrial BS channel, where the LoS dominates, provides the most advantageous channel conditions for mmWave communications. In contrast, the high altitude and mobility of UAVs is necessary to develop an efficient 3D beam-forming scheme for the mmWave UAV-terrestrial BS channel. The scheme selects the best beam to improve energy efficiency and maximize the transmission rate. The conventional schemes for mmWave 
beam selection are based on the estimation of real-time channel state information (CSI). Therefore, the UE is required to accurately estimate the channel and feed it back to the BS [10]. There is an exhaustive beam search [11], which is an easy and effective way for channel estimation, which finds the beam pair of transmit/receive that maximizes the signal-to-noise ratio (SNR) of the channel link, while the transmitter and receiver sequentially transmit the directional analog beams. However, this approach has been used for IEEE standard 802.11ad systems and 5G. This method is determined by the grid resolution and has a disadvantage of a large overhead because all transmission/reception beams must be sequentially transmitted. To overcome the overhead, a compressed sensing (CS)-based channel estimation algorithm based on the sparsity property of the millimeter wave channel was proposed [12], [13]. In addition, the beam search process can reduce the time by estimating the location or direction of the UE. The authors of [14] proposed an efficient beamforming and combining scheme using the exchange of location information among network nodes. In [15], a radar-based mmWave vehicle communication concept was introduced, and two iterative strategies for hybrid precoders and combiners were proposed in a vehicle-to-infrastructure (V2I) scenario. The simulation results confirmed that the radar and communication signals are similar to the arrival direction, so that the radar can provide useful information to configure the V2I link. The channel fingerprint can also act as useful historical information to aid beam selection. In [16], a multi-fingerprint-based beam selection was proposed and the fingerprints were collected for different traffic densities in a given location. The authors of [17] proposed a beam tracking scheme based on the channel fingerprint database, which comprises mappings between statistical beamforming gains and user locations.

This study proposes a coordinated mmWave beam selection scheme for cellular-connected UAVs. The proposed scheme configures a fingerprint database and performs coordinated beam selection. In the fingerprint database configuration, the best beam index from the serving cell and the interference beam indexes from neighboring cells are stored. In the coordinated beam selection, the best and interference beams are determined using the information of the fingerprint database instead of performing an exhaustive search, and the coordinated beam selection improves the SINR for aerial UEs. The remainder of this paper is organized as follows. Section 2 introduces system models for mmWave UAV communications. Section 3 describes the proposed coordinated beam selection based on fingerprints. Section 4 presents the simulation results. Finally, Section 5 concludes the paper.

\section{3GPP System Model}

\subsection{Cellular-connected UAV Communication}

We consider the NR downlink system to support both terrestrial and aerial UEs in a multi-cell environment, which is implemented by hexagonal cell and each cell comprises three sectors (Fig. 1). Terrestrial UEs are distributed outdoor with a height at $1.5 \mathrm{~m}$ and indoor in buildings with 4 to 9 floors [4], [18]. In contrast, aerial UEs are distributed outdoors with fixed height value chosen from $\{50,100,200,300\} \mathrm{m}$ [4].

Each terrestrial BS employs $N_{\mathrm{BS}}$ antennas and $N_{\mathrm{RF}} \mathrm{RF}$ chains to serve $K$ single-antenna UEs (including terrestrial and aerial UEs) [19], [20]. The received signal $y_{b k} \in \mathbb{C}$ of the $k$ th UE in the $b$ th cell is expressed by

$$
y_{b k}=\mathbf{h}_{b b k} \mathbf{w}_{b} \mathbf{s}_{b}+\sum_{i \neq b} \mathbf{h}_{i b k} \mathbf{w}_{i} \mathbf{s}_{i}+\epsilon_{b k},
$$


where $\mathbf{w}_{b} \in \mathbb{C}^{N_{\mathrm{BS}} \times K}$ is the digital precoding matrix, and $\boldsymbol{s}_{b} \in \mathbb{C}^{K \times 1}$ is the vector of the data symbols of cell $b$, i.e., the concatenation of each data stream vector of UE, such as $\boldsymbol{s}_{b}=$ $\left[s_{b, 1}, \ldots, s_{b, K}\right]^{T}$, where $s_{b, k}$ is the data symbol for UE $k$ of cell $b . \epsilon_{b k} \sim \mathcal{C N}\left(0, \sigma_{\epsilon}^{2}\right)$ is the thermal noise, and $\mathbf{h}_{i b k} \in \mathbb{C}^{1 \times N_{\mathrm{BS}}}$ denotes the channel vector between BS $i$ and UE $k$ in cell $b$.

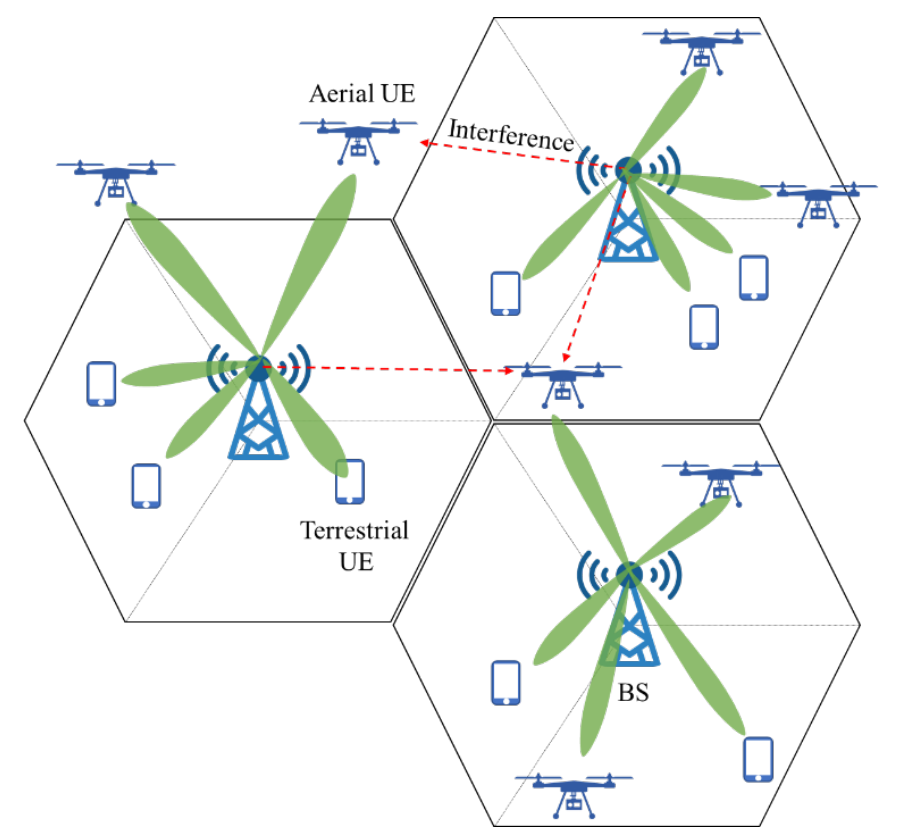

Fig. 1. Cellular networks for supporting terrestrial and aerial UEs. Each terrestrial BS employs $N_{\mathrm{BS}}$ antennas and $N_{\mathrm{RF}} \mathrm{RF}$ chains to serve $K$ single-antenna UEs simultaneously (including terrestrial and aerial UEs).

In the case of the existing massive MIMO system, one RF chain is required for each antenna; therefore, the antennas are arranged in a lens shape to reduce the number of RF chains. This lens antenna array serves as a spatial discrete Fourier transform (DFT) matrix containing the array steering vector [19], [20]. The spatial DFT codebook for uniform linear array (ULA) with $N_{\mathrm{BS}}$ antennas can be expressed as

$$
\mathbf{U}_{\mathrm{ULA}}\left(N_{\mathrm{BS}}\right)=\left[\mathbf{u}_{0}, \mathbf{u}_{1}, \ldots, \mathbf{u}_{N_{\mathrm{BS}}-1}\right],
$$

where the $b$ th codeword $\mathbf{u}_{b}$ is given by

$$
\mathbf{u}_{b}=\frac{1}{\sqrt{N_{\mathrm{BS}}}}\left[1 e^{j \frac{2 \pi}{N_{\mathrm{BS}}} b} e^{j \frac{2 \pi}{N_{\mathrm{BS}}} 2 b} \cdots e^{j \frac{2 \pi}{N_{\mathrm{BS}}}\left(N_{\mathrm{BS}}-1\right) b}\right]^{T} .
$$

ULA codebook can be extended to an uniform planer array (UPA) based on the Kronecker product. The codebook for UPA with $N_{\mathrm{BS}}\left(=N_{\mathrm{BS}}^{H} \times N_{\mathrm{BS}}^{V}\right)$ antennas can be expressed as

$$
\mathbf{U}_{\mathrm{UPA}}=\mathbf{U}_{\mathrm{ULA}}\left(N_{\mathrm{BS}}^{H}\right) \otimes \mathbf{U}_{\mathrm{ULA}}\left(N_{\mathrm{BS}}^{V}\right) \text {, }
$$

Then, the channel model in beamspace is represented as 


$$
\widetilde{\mathbf{H}}=\left[\tilde{\mathbf{h}}_{1}, \tilde{\mathbf{h}}_{2}, \ldots, \tilde{\mathbf{h}}_{K}\right]=\mathbf{U H}=\left[\mathbf{U h}_{1}, \mathbf{U h}_{2}, \ldots, \mathbf{U h}_{K}\right]
$$

where $\tilde{\mathbf{h}}_{k}, k=1,2, \ldots, K$ is the beamspace channel vector of the $k$ th UE. When the BS is equipped with $N_{\mathrm{RF}}=K \mathrm{RF}$ chains, a reduced-dimension matrix where $\widetilde{\mathbf{H}}_{b} \in \mathbb{C}^{K \times K}=$ $\widetilde{\mathbf{H}}(m,:)_{m \in \mathcal{S}}$ should be reconstructed, where $\mathcal{S}$ is the index set of the selected beams. We use a simple zero-forcing (ZF) precoder as a digital precoder. The reduced-dimension ZF precoding matrix $\widetilde{\mathbf{w}}_{b} \in \mathbb{C}^{K \times K}$ is expressed as

$$
\widetilde{\mathbf{w}}_{b}=\alpha \widetilde{\mathbf{H}}_{b}\left(\widetilde{\mathbf{H}}_{b}^{H} \widetilde{\mathbf{H}}_{b}\right)^{-1},
$$

where $\alpha$ is the scaling factor.

\subsection{Channel Model}

In 5G NR of 3GPP, a standard specification was developed channel model for the mmWave of $>6 \mathrm{GHz}$ [18] and aerial vehicle [4]. The channel model between the transmitter and receiver follows the spatial channel model (SCM). To express the propagation channel, the channel model uses stochastic and deterministic models. The former uses statistically representative model parameters through repeated measurements in a given propagation environment, and the latter uses a ray-tracing method that tracks channels by mathematically expressing the propagation environment between the transmitter and receiver. The statistical method is expressed as a probability function of a parameter that can represent the propagation environment, and the ray tracing method can accurately describe a specific propagation environment. 3GPP SCM is a geometry-based stochastic model that combines the above two methods, and it has two advantages.

We follow the procedure to generate a channel coefficient that comprises three major steps: general parameters, small-scale parameters, and coefficient generation [4], [18]. For general parameters, we set the scenario, network layout, and antenna parameters. Currently, the 3GPP standard specifies three scenarios for aerial vehicle, rural-macro, urban-macro, and urbanmicro. We consider the urban-macro scenario to determine channel parameters for the mmWave-UAV channel model. The large-scale fading data for urban macro (UMa) are listed in Table 1. Furthermore, the shadow fading has log-normal distribution. For small-scale fading, the channel impulse response between the receiving and transmitting antennas ( $u$ and $s$, respectively), corresponding to the time instant $t$ and delay $\tau$, can be expressed as follows.

$$
H_{u, s}(\tau, t)=\sqrt{\frac{1}{K_{R}+1}} H_{u, s}^{\mathrm{NLOS}}(\tau, t)+\sqrt{\frac{K_{R}}{K_{R}+1}} H_{u, s, 1}^{\mathrm{LOS}}(t) \delta\left(\tau-\tau_{1}\right),
$$

where $K_{R}$ denotes the Rician factor, and $H_{u, s}^{\mathrm{NLOS}}$ and $H_{u, s, 1}^{\mathrm{LOS}}$ represent the non-line-of-sight (NLoS) and LoS channel coefficients, respectively.

The main characteristics of UAV channels are summarized as follows: The LoS probability between the terrestrial BS and UAV increases as the height of the UAV increases. In particular, if the height of the UAV is more than $100 \mathrm{~m}$ in the UMa scenario, the channel condition among the BSs deployed in the network is always LoS. The path loss exponent of terrestrial-to-aerial links decreases as the height of the UAV increases. In fact, under LoS conditions, UAVs experience path loss similar to free-space propagation. When the terrestrial BS-UAV link is $\mathrm{LoS}$, the standard deviation of shadow fading decreases as the UAV height increases. In 
addition, there are three alternatives for small-scale fading [4]: 1) modification of the cluster delay line (CDL) channel model, 2) modification of large parameters (DS, ASA, ASD, ZSA, $\mathrm{ZSD}$, and $\mathrm{K}$ ), and 3) modification pertaining to $\mathrm{K}=15 \mathrm{~dB}$.

Table 1. Path loss and shadowing models for the UMa scenario [4], [18]

\begin{tabular}{|c|c|c|c|}
\hline $\begin{array}{c}\text { LoS/ } \\
\text { NLoS } \\
\text { condition }\end{array}$ & Pathloss [dB] & Std [dB] & $\begin{array}{c}\text { UE } \\
\text { height }\end{array}$ \\
\hline \multirow[t]{2}{*}{ LoS } & $P L_{L O S}= \begin{cases}28.0+22 \log _{10}\left(d_{3 D}\right)+20 \log _{10}\left(f_{c}\right), & 10 m \leq d_{2 D} \leq d_{B P}^{\prime} \\
28.0+40 \log _{10}\left(d_{3 D}\right)+20 \log _{10}\left(f_{c}\right) & d_{B P}^{\prime} \leq d_{2 D} \leq 5 k m \\
-9 \log _{10}\left(\left(d_{B P}^{\prime}\right)^{2}+\left(h_{B S}-h_{U E}\right)^{2}\right), & \end{cases}$ & $\sigma=4$ & $\begin{array}{l}1.5 m \leq h_{U E} \\
\leq 22.5 m\end{array}$ \\
\hline & $P L_{L O S}=28.0+22 \log _{10}\left(d_{3 D}\right)+20 \log _{10}\left(f_{c}\right)$ & $\begin{array}{l}\sigma=4.64 \exp \\
\left(-0.0066 h_{U E}\right)\end{array}$ & $\begin{array}{l}22.5 m<h_{U E} \\
\leq 300 m\end{array}$ \\
\hline \multirow{2}{*}{ NLoS } & $\begin{array}{l}P L_{\mathrm{NLOS}}=\max \left(P L_{\mathrm{LOS}}, P L_{\mathrm{NLOS}}^{\prime}\right) \\
P L_{\mathrm{NLOS}}^{\prime}=13.54+39.08 \log _{10}\left(d_{3 D}\right)+20 \log _{10}\left(f_{c}\right)-0.6\left(h_{U E}-1.5\right)\end{array}$ & \multirow{2}{*}{$\sigma=6$} & $\begin{array}{l}1.5 m \leq h_{U E} \\
\leq 22.5 m\end{array}$ \\
\hline & $P L_{\mathrm{NLOS}}=-17.5+\left(46-7 \log _{10}\left(h_{U E}\right)\right) \log _{10}\left(d_{3 D}\right)+20 \log _{10}\left(\frac{40 \pi f_{c}}{3}\right)$ & & $\begin{array}{l}22.5 m<h_{U E} \\
\leq 100 m\end{array}$ \\
\hline
\end{tabular}

\section{Coordinated Beam Selection Using Fingerprint}

In this section, we propose a coordinated beam selection using fingerprints for mmWave UAV communication spread over two phases: i) offline phase to build the fingerprint database and ii) online coordinated beam selection to mitigate the inter-cell interference for aerial UEs, which are explained below.

\subsection{Fingerprint Database Construction}

The fingerprint database for beam selection is constructed by placing the terrestrial and aerial UEs in each fingerprint location in each cell. In the $5 \mathrm{G}$ cellular system, beam selection comprises beam sweeping, beam measurement, beam determination, and beam reporting. Beam sweeping transmits a predefined set of beams to cover a spatial area. Among the $\mathrm{N}_{\mathrm{BS}}$ predefined beams, beam sweeping exhaustively searches for the optimal beam ID transmitted by the serving cell and the strongest interference beam ID caused by neighbor cells. The received signal at the UEs is measured, and the optimal beam at the UEs are selected according to the measurements obtained with the beam measurement procedure. If the UE is an aerial UE, the interfering cell ID and the corresponding interfering beam ID need to be measured and selected. Therefore, the optimal beam ID for UE $k$ in the $b$ th cell is selected from the codebook $\mathbf{U}$ using an exhaustive search [11].

$$
b_{k}^{*}=\underset{m \in\left\{1,2, \ldots, N_{B S}\right\}}{\operatorname{argmax}}\left|\mathbf{U}(m,:) \mathbf{h}_{b b k}\right|^{2} .
$$

Similarly, the strongest interference beam ID caused by the neighbor cell $i$ is obtained via the exhaustive search: 


$$
b_{k}^{i}=\underset{m \in\left\{1,2, \ldots, N_{B S}\right\}}{\operatorname{argmax}}\left|\mathbf{U}(m,:) \mathbf{h}_{i b k}\right|^{2} .
$$

Only aerial fingerprint locations are required to determine the strongest interference beam IDs. Then, the result is stored in the corresponding fingerprint database, i.e., all the fingerprint information within a cell coverage area.

The fingerprint database for each cell is divided into terrestrial and aerial fingerprint datasets. Table 2 shows the fingerprint database of the cell $b$, in which $P_{a}(a=1,2, \ldots, A)$ and $P_{t}(t=A+1, A+2, \ldots, A+T)$ are the aerial and terrestrial fingerprint locations, respectively. For the location of aerial and terrestrial UEs, we express location by using the 3D Cartesian coordinate system. Each UE is represented by $P=(x, y, z)$.

$I_{-} I D_{i}(i=1,2)$ is an interference cell ID for a fingerprint location, $b_{t}^{*}$ is the optimal beam ID for $P_{t}$, and $b_{a}^{*}$ and $\tilde{b}_{a}^{i}$ are the optimal and the strongest interference beam IDs, respectively, corresponding to the interference cell $i$ for $P_{a}$. The number of interference cells is two ( $i=$ 1,2 ) because we consider the intra-site joint transmission (JT) for aerial UEs, which will be discussed in the following subsections:

Table 2. Example of fingerprint for cell $b$

\begin{tabular}{|c|c|c|c|c|c|c|c|c|c|c|}
\hline \multicolumn{7}{|c|}{ Aerial UE } & \multicolumn{4}{|c|}{ Terrestrial UE } \\
\hline \multicolumn{2}{|c|}{$P_{1}$} & \multicolumn{2}{|c|}{$P_{2}$} & $\ldots$ & \multicolumn{2}{|c|}{$P_{A}$} & $P_{A+1}$ & $P_{A+2}$ & $\ldots$ & $P_{A+T}$ \\
\hline \multicolumn{2}{|c|}{$b_{1}^{*}$} & \multicolumn{2}{|c|}{$b_{2}^{*}$} & & \multicolumn{2}{|c|}{$b_{A}^{*}$} & \multirow{3}{*}{$b_{A+1}^{*}$} & \multirow{3}{*}{$b_{A+2}^{*}$} & \multirow{3}{*}{$\ldots$} & \multirow{3}{*}{$b_{A+T}^{*}$} \\
\hline$I_{-} I D_{1}$ & $\tilde{b}_{1}^{1}$ & $I_{-} I D_{1}$ & $\tilde{b}_{2}^{1}$ & $\cdots$ & $I_{-} I D_{1}$ & $\tilde{b}_{A}^{1}$ & & & & \\
\hline$I_{-} I D_{2}$ & $\tilde{b}_{1}^{2}$ & $I_{-} I D_{2}$ & $\tilde{b}_{2}^{2}$ & & $I_{-} I D_{2}$ & $\tilde{b}_{A}^{2}$ & & & & \\
\hline
\end{tabular}

\subsection{Cooperative beam for aerial UE}

As location information can be used with a built-in global navigation satellite system or a network positioning system, we assume that the UE currently knows its location. Therefore, the UE measures its location and transmits it to the BS. Then, the BS searches the fingerprint database for the current location fed back from the UE. In the search process, the serving BS, which has received the location feedback from the UE, searches and compares the location in the fingerprint database one by one. Location search refers to finding the fingerprint location closest to the current location of the UE (i.e., the smallest error value) in the fingerprint database, and the best-matched location of the UE $k$ is as follows.

$$
P_{k}^{*}=\underset{i \in 1,2, \ldots, T+A}{\operatorname{argmin}}\left|P_{k}-P_{i}\right|^{2},
$$

where $P_{k}$ is the current location of the UE $k, P_{i}$ is the fingerprint location $i$, and $P_{k}^{*}$ is the closest fingerprint location. As a result, we can obtain a fingerprint location most closely matched with the current location of the UE.

After the search process, the BS can determine the terrestrial or aerial UE through the bestmatched fingerprint location. If the best-matched fingerprint is in the aerial fingerprint location, the UE can be identified as an aerial UE; otherwise, it is a terrestrial UE. When the UE is terrestrial, the serving BS transmits the optimal beam to the UE according to the beam ID, corresponding to the best-matched fingerprint location. The aerial UE receives interference from more cells in the downlink because the aerial UE has LoS propagation conditions for more cells with a higher probability compared with the terrestrial UE. Therefore, we apply JT to improve the SINR performance by reducing interference from terrestrial BS for aerial UE. 
In this study, multiple cells belonging to the same site are coordinated, and data are jointly transmitted to the UEs. As intra-site JT-coordinated multi-point is already supported in 3GPP standardization, enhancements are not required. With the beam cooperation for aerial UE, the interference from the neighboring cells is reduced because a neighboring cell is converted from the interference signal to the desired signal. This advantage can be observed from the SINR expression. Assuming that the UEs have perfect CSI, the resulting instantaneous SINR with the cooperative beam at aerial UE $k$ in cell $b$ is

$$
S I N R_{\text {coop }}=\frac{\sum_{c \in \mathcal{C}} 10^{\xi_{c} / 10}}{\left(\sum_{j \in \mathcal{B}} 10^{\xi_{j} / 10}\right)-\sum_{c \in \mathcal{C}} 10^{\frac{\xi_{c}}{10}+N_{0}}},
$$

where $\xi_{j}$ is the received power of $\mathrm{UE} k$ from cell $j$, and $\mathcal{B}$ and $\mathcal{C}$ represent a set of BSs and JT BSs, respectively. In contrast, the SINR without beam cooperation at terrestrial UE $k$ in cell $b$ is

$$
\operatorname{SINR}_{\text {non-coop }}=\frac{10^{\xi_{b} / 10}}{\left(\sum_{j \in \mathcal{B}} 10^{\xi_{j} / 10}\right)-10^{\xi_{b} / 10}+N_{0}} .
$$

Thus, the cooperative beam not only improves the received power but also reduces the interference power.

\section{Simulation Results}

\subsection{Simulation model and parameters}

Simulation parameters are based on a bandwidth of $100 \mathrm{MHz}$ with a carrier frequency of 30 GHz in the 3GPP NR downlink system. For the UMa scenario, a cell layout comprising 19 cell sites with 3 sectors per site is deployed, and the number of UEs (including both aerial and terrestrial UEs) per sector is assumed to be 15 . To evaluate the effect of aerial UEs with different ratios in the sector, the values of $0,0.1,1,3$, and 5 were considered. The terrestrial UEs were on the terrestrial buildings and inside the buildings. The height of the aerial UE is randomly selected from the values of 50,100, 200, and $300 \mathrm{~m}$. In contrast, an aerial UE is modeled as an outdoor UE of height greater than the terrestrial UE. The specific parameters are listed in Table 3.

Table 3. Simulation parameters

\begin{tabular}{|l|l|}
\hline Parameters & Assumptions \\
\hline \hline Cell Layout & Hexagonal grid, 19 cell sites, 3 sectors/site \\
\hline Center Frequency & $30 \mathrm{GHz}$ \\
\hline Bandwidth & $100 \mathrm{MHz}$ \\
\hline Scenario & UMa \\
\hline $\begin{array}{l}\text { No. of UEs per Sector } \\
\left(N_{\text {terrestrial }}+N_{\text {aerial }}\right)\end{array}$ & $15 \mathrm{UEs}$ \\
\hline $\begin{array}{l}\text { Aerial UE ratio } \\
\left(N_{\text {aerial }} / N_{\text {terrestrial }}\right)\end{array}$ & $\begin{array}{l}\text { Case } 1: 0 \%\left(\text { corresponding to } N_{\text {aerial }}=0\right) \\
\text { Case } 2: 0.67 \%\left(\text { corresponding to } N_{\text {aerial }}=0.1\right) \\
\text { Case } 3: 7.1 \%\left(\text { corresponding to } N_{\text {aerial }}=1\right) \\
\text { Case } 4: 25 \%\left(\text { corresponding to } N_{\text {aerial }}=3\right) \\
\text { Case } 5: 50 \%\left(\text { corresponding to } N_{\text {aerial }}=5\right)\end{array}$ \\
\hline
\end{tabular}




\begin{tabular}{|l|l|}
\hline \multirow{2}{*}{ Antenna Configuration } & $\mathrm{BS}: N_{\mathrm{BS}}^{H}=N_{\mathrm{BS}}^{V}=8, N_{B S}=64$ \\
\cline { 2 - 3 } & $\mathrm{UE}:$ Single antenna \\
\hline BS Max TX Power & $35 \mathrm{dBm}$ \\
\hline Scheduling & Round Robin \\
\hline Traffic Model & Full Buffer \\
\hline Antenna vertical pattern (dB) & $A_{E, V}\left(\theta^{\prime \prime}\right)=-\min \left\{12\left(\frac{\theta^{\prime \prime}-90^{\circ}}{\theta_{3 \mathrm{~dB}}}\right)^{2}, S L A_{V}\right\}, \theta_{3 \mathrm{~dB}}=65^{\circ}, S L A_{V}=30 \mathrm{~dB}$ \\
\hline Antenna horizontal pattern (dB) & $A_{E, H}\left(\phi^{\prime \prime}\right)=-\min \left\{12\left(\frac{\phi^{\prime \prime}}{\phi_{3 \mathrm{~dB}}}\right)^{2}, A_{m}\right\}, \phi_{3 \mathrm{~dB}}=65^{\circ}, A_{m}=30 d B$ \\
\hline $\begin{array}{l}\text { Combining method for 3D } \\
\text { antenna pattern }(\mathrm{dB})\end{array}$ & $\mathrm{A}^{\prime \prime}\left(\theta^{\prime \prime}, \phi^{\prime \prime}\right)=-\min \left\{-\left[\mathrm{A}_{\mathrm{E}, \mathrm{V}}\left(\theta^{\prime \prime}\right)+\mathrm{A}_{\mathrm{E}, \mathrm{H}}\left(\phi^{\prime \prime}\right)\right], \mathrm{A}_{\mathrm{m}}\right\}$ \\
\hline Antenna gain & $8 \mathrm{dBi}$ \\
\hline UE Noise Figure & $9 \mathrm{~dB}$ \\
\hline
\end{tabular}

\subsection{UAV Effect}

Fig. 2 shows the cumulative distribution function (CDF) of the SINR for aerial and terrestrial UEs for various aerial UE ratios. In the downlink, the aerial UE receives interference from more cells. Therefore, compared with the SINR of case 1 (no aerial UE presence), case 5 (aerial UE ratio is $50 \%$ ) has an average performance degradation of $-3.5 \mathrm{~dB}$. In addition, the SINR of the terrestrial UE is not affected by the aerial UE. However, as more resources have to be allocated to the aerial UE to compensate for the low SINR of the aerial UE, the cellular system suffers from the overall performance degradation. Consequently, the downlink performance of terrestrial UEs is affected by the aerial devices.

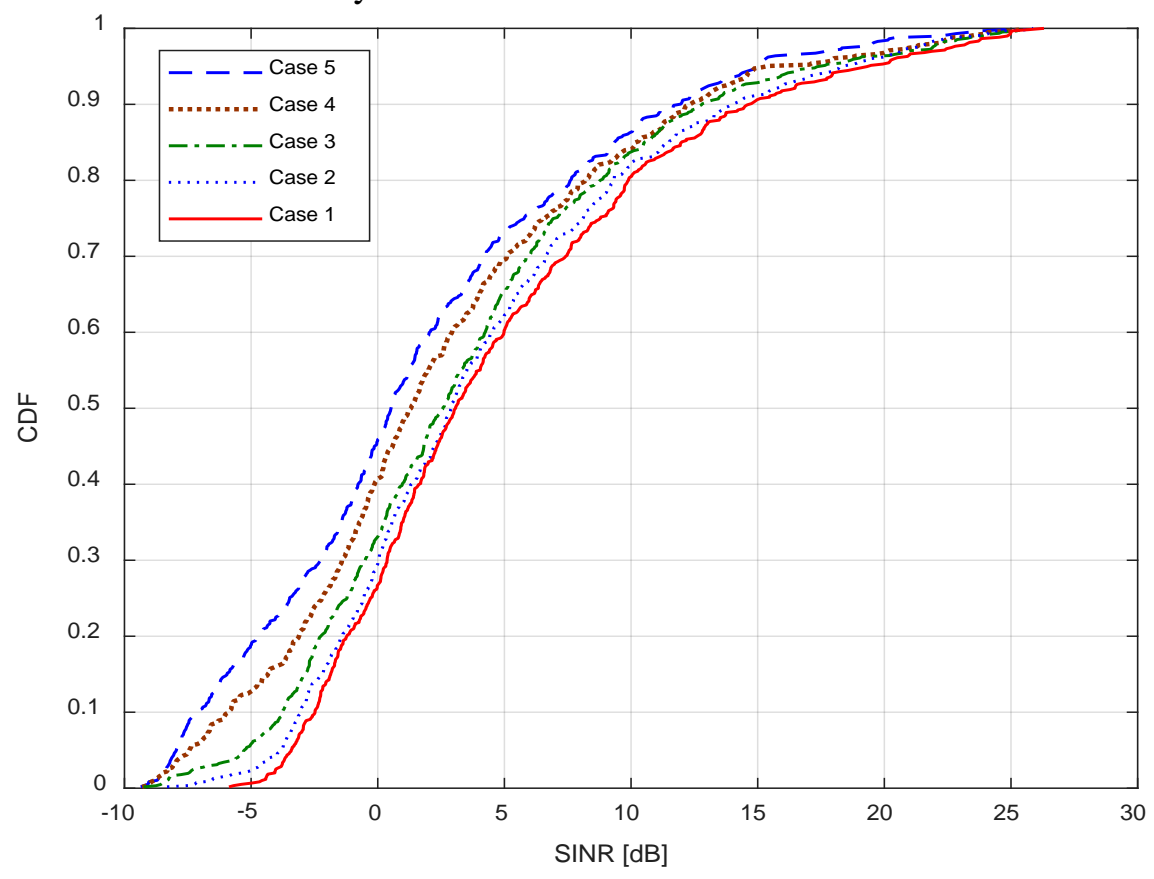

Fig. 2. CDF of the SINR versus the aerial UE ratio. 
Fig. 3 shows the CDF of the SINR versus the aerial UE height (50, 100, 200, and $300 \mathrm{~m}$ ). These SINR values are obtained by plotting five UAVs per sector. It shows that the performance of the downlink channel deteriorates as the height of the aerial UE increases from $50 \mathrm{~m}$ to the maximum flight altitude of $300 \mathrm{~m}$. As the height of the aerial UE increases, the probability that the aerial UE receives LoS signals from several BSs increases [4]. Thus, the aerial UE has reduced path loss with the serving BS but does not compensate for the additional interference received from a significant number of BSs.

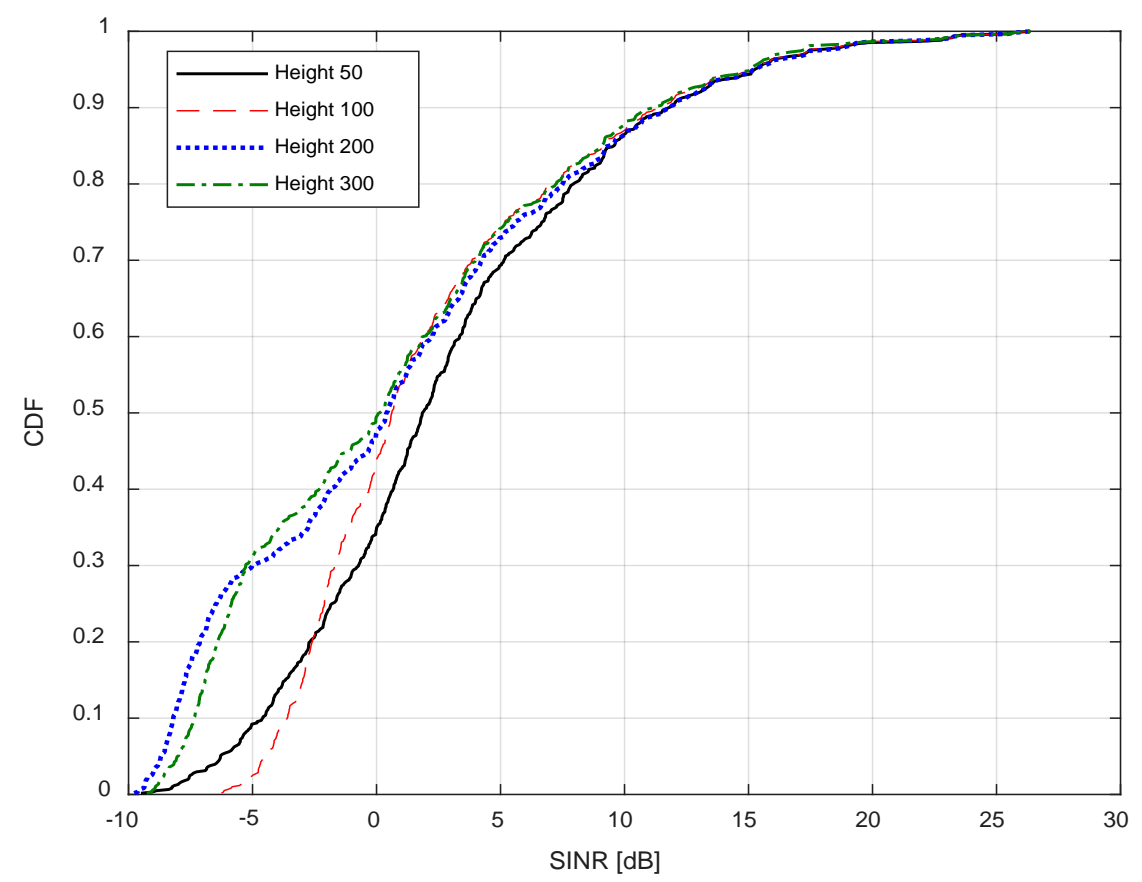

Fig. 3. CDF of the SINR versus the aerial UE height.

\subsection{Performance of Cooperative Beam}

To evaluate the performance of the proposed scheme, we compare and analyze the following schemes:

(1) Random beam selection (R-BS): Each UE is served with its serving cell, and beams are randomly selected.

(2) Exhaustive search beam selection (ES-BS): Each UE is served with its serving cell, and beams are selected via an exhaustive search.

(3) Beam selection with single-cell transmission (SC-BS): Each UE is served with its serving cell, and fingerprint-based beam selection is used.

(4) Beam selection with JT (JT-BS): It is same as scheme (3), but intra-site JT is used for aerial UEs.

Fig. 4 plots the CDF of the SINR for schemes (1)-(4), where the aerial UE ratio is 50\% (five aerial UEs per cell). The proposed JT-BS can achieve a higher SINR than the other schemes. This is because aerial UEs have a low SINR value owing to the inter-cell interference. The performance of schemes (3) and (4) is significantly affected by the accuracy of the fingerprint search process of the UE. When the difference in error between the fingerprint and actual location of the UE is large, the matching result of the BS will be incorrect, and the 
selected beam is not the best beam for the current UE. The fingerprint-based scheme degrades performance owing to location errors but can avoid the large overhead caused by the beam sweeping. In the fingerprint-based beam selection schemes, the UE only needs to feed back the current location, and the BS matches the current location of the UE with the fingerprint database to obtain the optimal/interference beam.

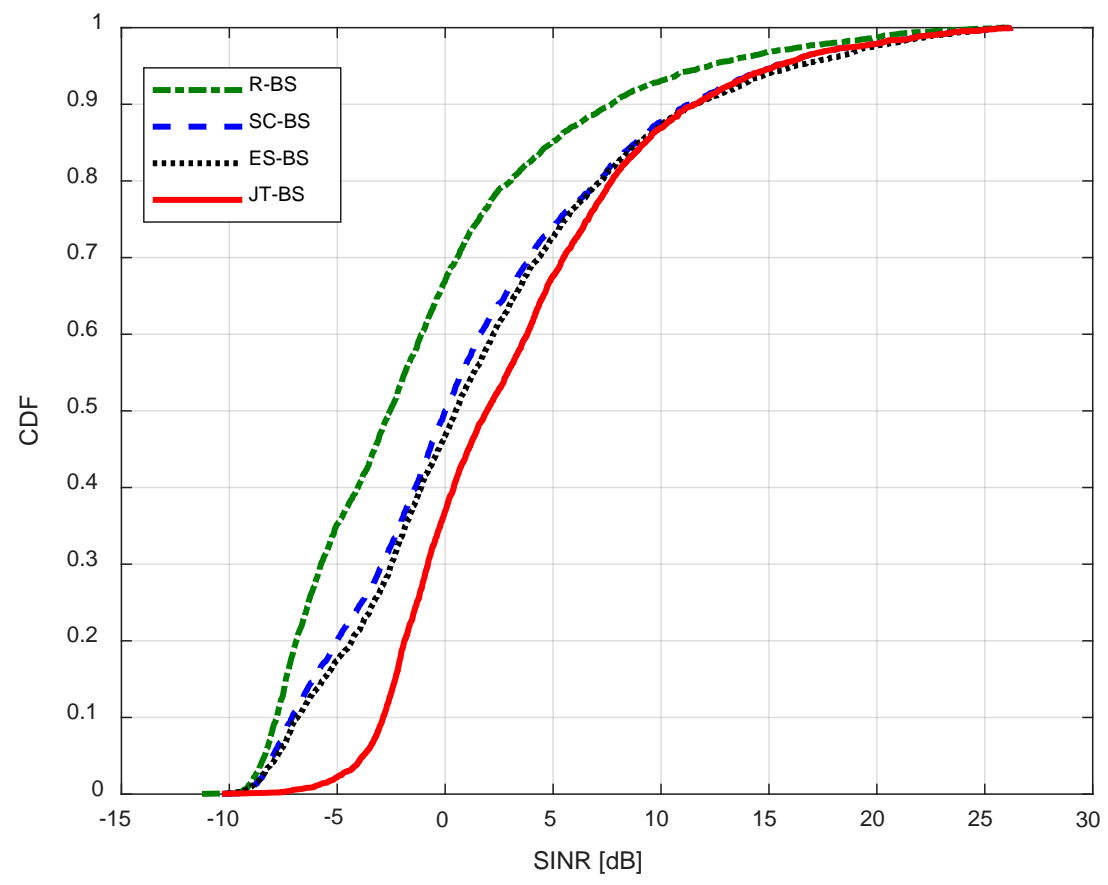

Fig. 4. CDF of the SINR for schemes (3) and (4). In this figure, we consider the $7.1 \%$ and $50 \%$ aerial UE ratios (1 and 5 aerial UEs per cell, respectively).

Fig. 5 and Fig. 6 plot the CDF of the SINR and the spectral efficiency for schemes (3) and (4) in cellular-connected UAV communications when the aerial UE ratios are $7.1 \%$ and $50 \%$. As the aerial UEs experience LoS propagation conditions for more cells with a higher probability, in comparison with terrestrial UEs, aerial UEs receive interference from more cells in the downlink. Therefore, as the number of aerial UE increases, interference from neighbor cells increases for scheme (3). In addition, the SINR is improved by changing the interference signal into the desired signal through the beam cooperation of scheme (4). When the aerial UE ratio is $7.1 \%$, there is a negligible difference in performance between techniques 3 and 4 . This is because the possibility of inter-cell beam interference of a smaller number of aerial UEs is very small; therefore, the difference in performance between the two schemes is not large. Therefore, the proposed scheme (JT-BS) improves SINR and spectrum efficiency performance by effectively reducing inter-cell interference when the ratio of public UEs is high. 


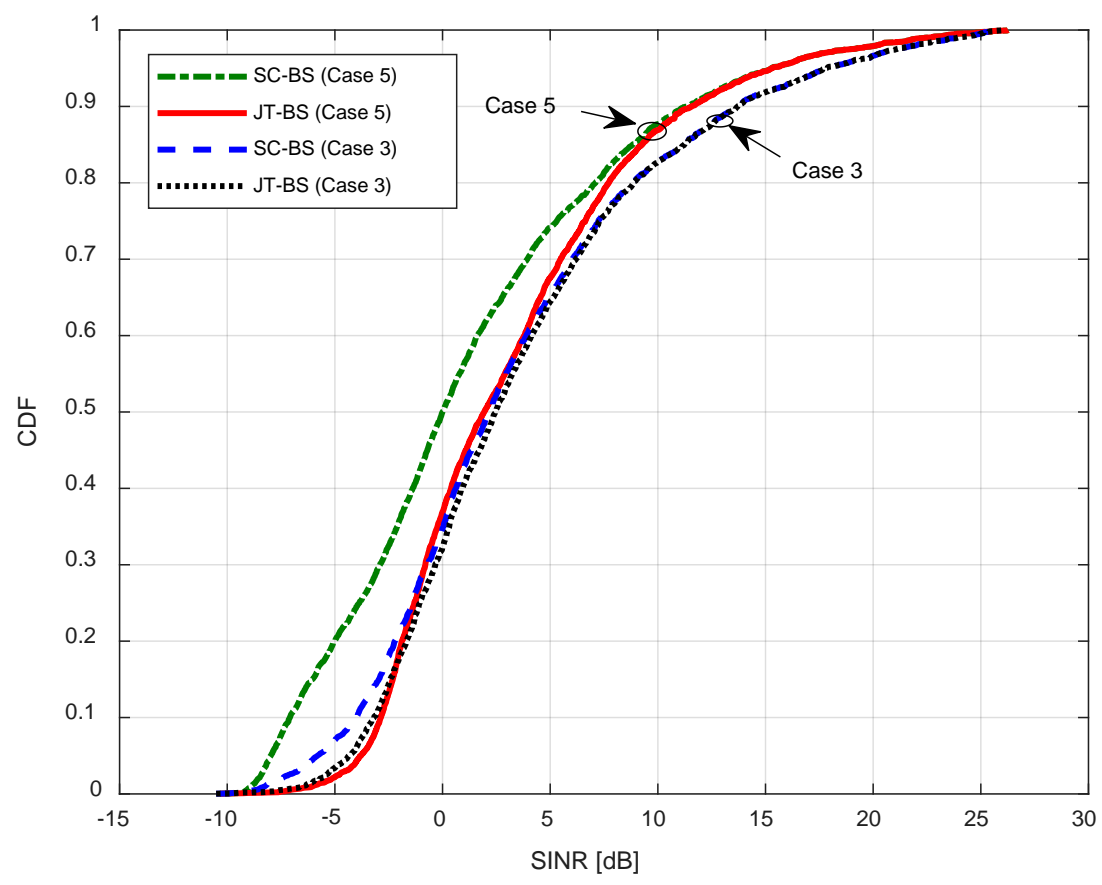

Fig. 5. CDF of the SINR for schemes (3) and (4). In this figure, we consider the $7.1 \%$ and $50 \%$ aerial UE ratios (1 and 5 aerial UEs per cell, respectively).

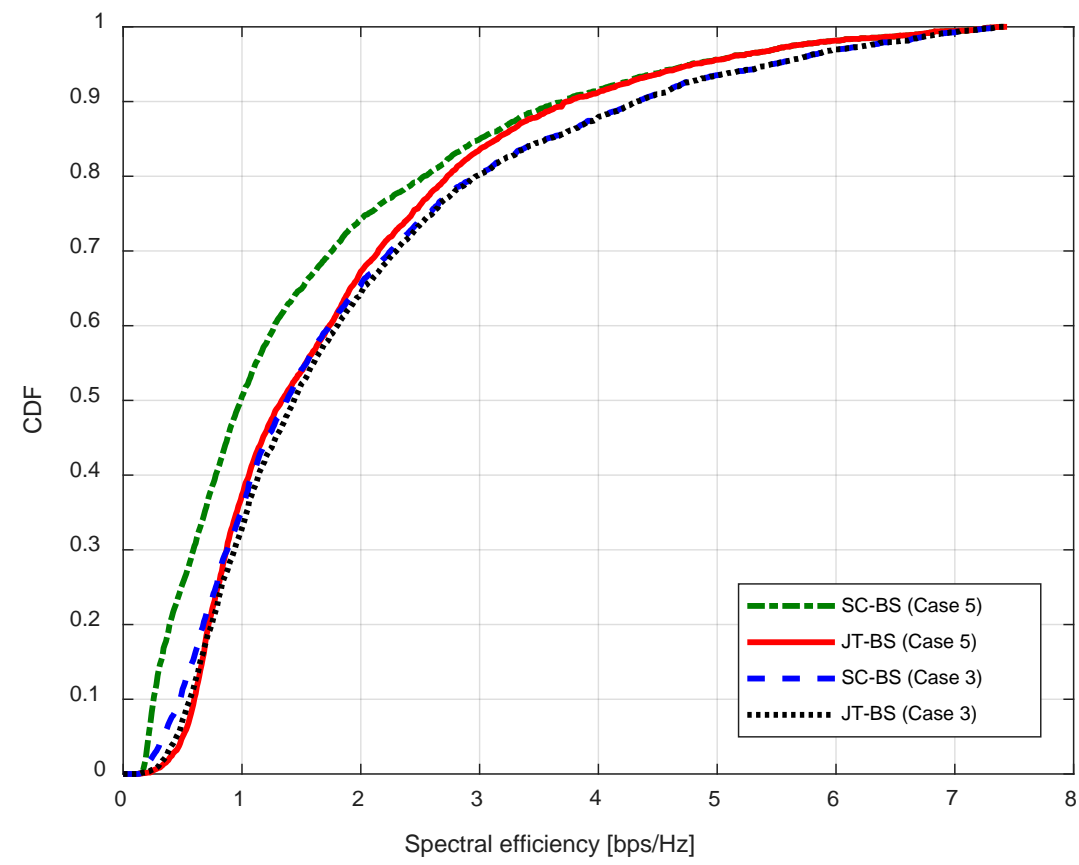

Fig. 6. CDF of the spectral efficiency for schemes (3) and (4). In this figure, we consider the $7.1 \%$ and $50 \%$ aerial UE ratios ( 1 and 5 aerial UEs per cell, respectively). 


\section{Conclusion}

This study proposed a fingerprint-based beam selection and cooperation scheme for mmWave UAV communications. The proposed scheme constructs an offline fingerprint database for beam selection and performs online beam cooperation. In the offline phase, the best beam index from serving cells and the interference beam indexes from neighboring cells are stored. In the online phase, the best and interference beams are determined using the fingerprint database instead of an exhaustive search, and the beam cooperation is performed to improve SINR for aerial UEs. System-level simulations are performed to assess the UAV effect based on the 3GPP new radio mmWave and UAV channel models. The simulation results showed that the proposed beam selection scheme can reduce the beam sweeping overhead and intercell interference. For future work, we will study a deep learning-based beam selection using constructed fingerprint database as training data. We will also collect other side-information such as UE's speed, direction, and traffic density to improve the performance of beam selection.

\section{References}

[1] Q. Zeng and Z. Zhang, “The Full-Duplex Device-to-Device Security Communication Under the Coverage of Unmanned Aerial Vehicle,” KSII Trans. Internet Inf. Syst., vol. 13, no. 4, pp. 19411960, April 2019. Article (CrossRef Link)

[2] Q. Zeng, W. Huangfu and T. Liu, "Power Allocation and Mode Selection in Unmanned Aerial Vehicle Relay Based Wireless Networks,” KSII Trans. Internet Inf. Syst., vol. 13, no. 2, pp. 711732, Feb. 2019. Article (CrossRef Link)

[3] FAA, "Federal Aviation Administration; Aerospace Forecasts Fiscal Years 2018-2038," [Online]. Available: $\quad$ https://www.faa.gov/data_research/aviation/aerospace_forecasts/media/FY201838_FAA_Aerospace_Forecast.pdf.

[4] 3GPP, “3rd Generation Partnership Project; Enhanced LTE support for aerial vehicles,” TR 36.777, V15.0.0, Jan. 2018. [Online]. Available: https://portal.3gpp.org/desktopmodules/Specifications/SpecificationDetails.aspx?specificationId $=3231$

[5] B. V. D. Bergh, A. Chiumento, and S. Pollin, "LTE in the sky: Trading off propagation benefits with interference costs for aerial nodes,” IEEE Commun. Mag., vol. 54, no. 5, pp. 44-50, May 2016. Article (CrossRef Link)

[6] X. Lin et al., "The sky is not the limit: LTE for unmanned aerial vehicles," IEEE Commun. Mag., vol. 56, no. 4, pp. 204-210, Apr. 2018. Article (CrossRef Link)

[7] Y. Zeng, J. Lyu, and R. Zhang, “Cellular-connected UAVs: Potentials, challenges and promising technologies,” IEEE Wireless Commun., vol. 26, no. 1, pp. 120-127, Feb. 2019.

Article (CrossRef Link)

[8] R. Amorim, H. Nguyen, P. Mogensen, I. Z. Kovács, J. Wigard and T. B. Sørensen, "Radio channel modeling for UAV communication over cellular networks,” IEEE Wireless Commun. Lett., vol. 6, no. 4, pp. 514-517, Aug. 2017. Article (CrossRef Link)

[9] Z. Xiao, P. Xia, and X.-G. Xia, "Enabling UAV cellular with millimeter-wave communication: potentials and approaches,” IEEE Commun. Mag., vol. 54, no. 5, pp. 66-73, May 2016. Article (CrossRef Link)

[10] A. Alkhateeb, G. Leus and R. W. Heath, "Limited feedback hybrid precoding for multi-user millimeter wave systems,” IEEE Trans. Wireless Commun., vol. 14, no. 11, pp. 6481-6494, Nov. 2015. Article (CrossRef Link)

[11] Junyi Wang et al., "Beam codebook based beamforming protocol for multi-Gbps millimeter-wave WPAN systems,” IEEE J. Sel. Areas Commun., vol. 27, no. 8, pp. 1390-1399, Oct. 2009. Article (CrossRef Link) 
[12] A. Alkhateeb, O. El Ayach, G. Leus and R. W. Heath, "Channel estimation and hybrid precoding for millimeter wave cellular systems,” IEEE J. Sel. Top. Signal Process., vol. 8, no. 5, pp. 831846, Oct. 2014. Article (CrossRef Link)

[13] G. C. Alexandropoulos and S. Chouvardas, "Low complexity channel estimation for millimeter wave systems with hybrid A/D antenna processing," in Proc. of IEEE Global Commun. Conf. Workshops (GC Wkshps), Dec. 2016. Article (CrossRef Link)

[14] G. C. Alexandropoulos, "Position aided beam alignment for millimeter wave backhaul systems with large phased arrays," in Proc. of IEEE Int. Workshop on Computational Advances in MultiSensor Adaptive Processing (CAMSAP), Dec. 2017. Article (CrossRef Link)

[15] N. González-Prelcic, R. Méndez-Rial and R. W. Heath, "Radar aided mmWave beam alignment in V2I communications supporting antenna diversity," in Proc. of Information Theory and Applications Workshop (ITA), Feb. 2016. Article (CrossRef Link)

[16] K. Satyanarayana et al., "Deep Learning Aided Fingerprint-Based Beam Alignment for mmWave Vehicular Communication,” IEEE Trans. Veh. Technol., vol. 68, no. 11, pp. 10858-10871, Nov. 2019. Article (CrossRef Link)

[17] R. Deng et al., "Channel Fingerprint Based Beam Tracking for Millimeter Wave Communications," IEEE Commun. Lett., vol. 24, no. 3, pp. 639-643, March 2020. Article (CrossRef Link)

[18] 3GPP, "3rd Generation Partnership Project; Study on channel model for frequencies from 0.5 to 100 GHz,” TR 38.901, V16.1.0, Jan. 2020. [Online] Available: https://portal.3gpp.org/desktopmodules/Specifications/SpecificationDetails.aspx?specificationId $=3173$

[19] A. Sayeed and J. Brady, "Beamspace MIMO for high-dimensional multiuser communication at millimeter-wave frequencies," in Proc. of IEEE Global Commun. Conf. Workshops (GC Wkshps), Dec. 2013. Article (CrossRef Link)

[20] P. V. Amadori and C. Masouros, "Low RF-complexity millimeter-wave beamspace-MIMO systems by beam selection,” IEEE Trans. Commun., vol. 63, no. 6, pp. 2212-2223, June 2015. Article (CrossRef Link) 

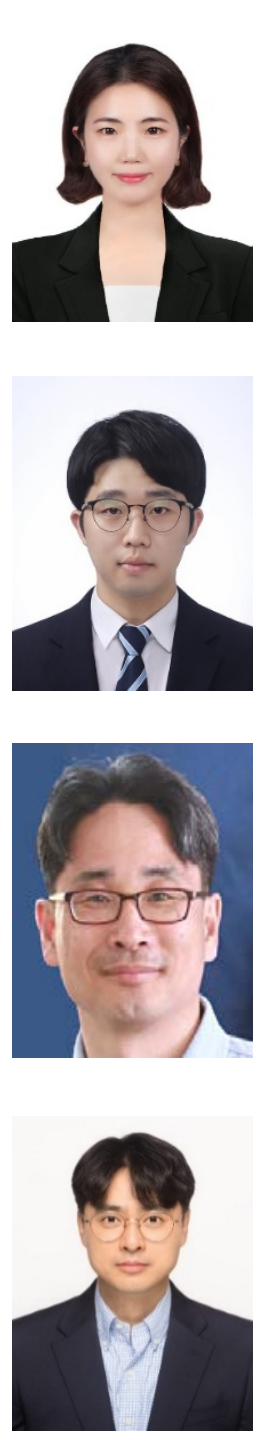

Cheol Hong Kim received the B.S. degree in Computer Engineering from Seoul National University, Seoul, Korea in 1998 and M.S. degree in 2000. He received the Ph.D. in Electrical and Computer Engineering from Seoul National University in 2006. He worked as a senior engineer for SoC Laboratory in Samsung Electronics, Korea from Dec. 2005 to Jan. 2007. He also worked as a Professor at Chonnam National University, Korea from 2007 to 2020. Now he is working as a Professor at School of Computer Science and Engineering, Soongsil University, Korea. His research interests include computer systems, embedded systems, mobile systems, computer architecture, low power systems, and intelligent computer systems.

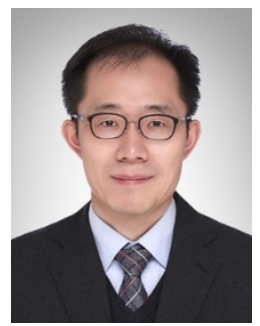

Intae Hwang received a B.S. degree in Electronics Engineering from Chonnam National University, Gwangju, Korea in 1990 and a M.S. degree in Electronics Engineering from Yonsei University, Seoul, Korea in 1992, and a Ph.D. degree in Electrical \& Electronics Engineering from Yonsei University, Seoul, Korea in 2004. He was a senior engineer at LG Electronics from 1992 to 2005. He is currently a Professor in the Department of Electronic Engineering and Department of ICT Convergence System Engineering at Chonnam National University, Gwangju, Korea from 2006. His research interests include digital communication, wireless communication system, and next-generation mobile communication: MIMO-OFDM, V2X, NR-MIMO, NTN, and artificial intelligence. 